\title{
Mode Structure of Disruption Precursors in TFTR Enhanced Reversed Shear Discharges
}

\author{
M. Okabayashi, E. Fredrickson, J. Manickam, G. Taylor, \\ S. Batha ${ }^{1}$, M. Bell, R. Budny, B. LeBlanc, F. Levinton ${ }^{1}$, \\ J. Jackson ${ }^{2}$, K. McGuire, R. Nazikian and M. Zarnstorff \\ Princeton Plasma Physics Laboratory, Princeton, N.J USA \\ ${ }^{1}$ Fusion Physics and Technology, Torrance, CA USA \\ ${ }^{2}$ Southern University A\&M College, Baton Rouge, LA USA
}

\begin{abstract}
The mode structure of the disruption precursors in the TFTR enhanced reversed shear parameters has been studied by using $T_{e}$ fluctuation profiles and $q$ profiles obtained from Electron Cyclotron Emission(ECE) and Motional Stark Effect(MSE) measurements. The observed profiles of the radial displacement associated with the MHD modes were consistent with the displacement profiles expected from the ideal MHD external kink mode. The observed mode frequencies differ from the plasma toroidal rotation frequency measured with CVI charge-exchange recombination light. The independence of the mode frequency from the plasma rotation frequency supports the ideal MHD hypothesis. Possible causes of the frequency difference are discussed.
\end{abstract}

\section{Introduction}

From the beginning of fusion research, ideal MHD theory has been the guiding principle for achieving quiescent plasmas. The designs of many magnetic fusion devices from mirrors to toroidal devices have been based primarily on predictions from ideal MHD theory. In the past few decades, the tokamak approach has made remarkable progress in improving operational techniques, and achieving extremely collisionless plasmas with high electron and ion temperatures. One significant limitation in tokamak operation, however, is the occurrence of disruptions, plasma termination events which occur on a very rapid (Alfvénic) time scale. A leading candidate for the cause of disruptions is the ideal kink instability.

Qualitatively, ideal MHD theory and its predictions have been consistent with many experimental observations. In particular, the upper limit of achievable beta, derived from marginal ideal MHD stability analysis, has shown qualitative agreement with observations in various tokamak devices 
$[1,2,3,4,5,6,7]$. However, ideal MHD theory is not yet completely successful. An example is the understanding of the sawtooth behavior [8, 9, 10]. The MHD activity predicted near the central core is sensitive to the $q$ value at the axis and the magnetic shear $q^{\prime} / q$ at the surface where $q=1$ so that the validity of ideal MHD theory near the core still requires further experimental and theoretical investigations.

According to ideal MHD theory, the reversed shear configuration is a promising approach for achieving high $\beta$ in tokamaks by stabilizing the most dangerous high-n ballooning modes. Furthermore, the bootstrap current created by the local pressure gradient near the axis can sustain self-consistently the reverse shear configuration, leading to a steady state tokamak regime. Several experiments have achieved reversed shear configurations with improved transport properties $[11,12,13,14,15,16]$. In the TFTR experiments, the reversed shear configuration has been explored by transiently creating a hollow current profile early in the discharge with a current ramp technique [11]. The hollow current profile is maintained by freezing the poloidal and toroidal flux with NBI heating. When the inverted $q$-profile discharge evolves in time, the minimum q value, qmin, gradually decreases toward 2. Resistive tearing modes are excited with qedge approaching integers and a major disruption takes place, if the programming is not carefully tailored [12].

The high $\beta$ disruptions in TFTR have been studied with various configurations $[11,17,18,19,20]$. In these studies, the consistency with ideal MHD predictions has been the central issue. For the non-reversed shear configuration, the observations of fast growth rates and broad radial widths of the modes suggested that the onset of global external kink is a most likely candidate for the initiation of the disruption process $[17,18]$. An absence of any indication of island formation also supported the ideal MHD hypothesis. The mode structures derived from Electron Cyclotron Emission(ECE) and soft $\mathrm{x}-$ ray arrays were compared with theoretical predictions from ideal MHD theory. However, in the early studies, an independent q-measurement was not available. A major result from these studies was that the coupling of the external kink with ballooning modes just before the onset of disruption was observed $[19,20]$.

The present analysis is intended to provide a quantitative study of disruption precursors in the TFTR reversed shear configuration. Two parameter regimes have been explored in TFTR, one with smaller $\mathrm{r}_{\mathrm{q} \cdot \min },\left(\mathrm{r}_{\mathrm{q} \cdot \mathrm{min}} / \mathrm{a}=\right.$ $0.4-0.5)$ with $\mathrm{I}_{\mathrm{p}}=1.6 \mathrm{MA}$ and, the other with $\mathrm{I}_{\mathrm{p}}=2.2 \mathrm{MA}$ and larger $\mathrm{r}_{\mathrm{q} \cdot \min },\left(\mathrm{r}_{\mathrm{q} \cdot \min } / \mathrm{a}=0.6\right)$. Here, $\mathrm{r}_{\mathrm{q} \cdot \min }$ is the distance of the minimum q location from the magnetic axis, and a is the distance from the magnetic axis to the outboard plasma edge. In both cases, the $q_{\min }$ approaches 2 in the interval before a major disruption. The main parameters for the plasma studied in this analysis are listed in Table-I. Here $\beta_{\mathrm{p}}$ is $\beta$-poloidal and $\beta_{\mathrm{n}}=\beta /\left(\mathrm{a} \mathrm{I}_{\mathrm{p}} / \mathrm{B}_{\mathrm{t}}\right)$ is 
the Troyon-normalized $\beta$. The present discussion includes one case of steady state MHD activity for comparison purpose.

\section{Table -I}

\begin{tabular}{|c|c|c|c|c|}
\hline & small & small & small & large \\
\hline & $\mathrm{r}_{\mathrm{q} \cdot \min }$ & $r_{q \cdot \min }$ & $r_{q \cdot \min }$ & $r_{q \cdot \min }$ \\
\hline shot & $\sharp 84011$ & $\sharp 84011$ & $\sharp 91243$ & $\sharp 93260$ \\
\hline time & $2.8 \mathrm{sec}$ & $3.08 \mathrm{sec}$ & 3.135 & $2.497 \mathrm{sec}$ \\
\hline$\tau_{\text {growth }}$ & steady state & $\simeq 150 \mu \mathrm{sec}$ & $\simeq 120 \mu \mathrm{sec}$ & $\simeq 150 \mu \mathrm{sec}$ \\
\hline$q_{\min }$ & 2.3 & 2.0 & 2.0 & 2.2 \\
\hline qedge & 5.2 & 4.8 & 6.2 & 4.4 \\
\hline $\mathrm{r}_{\mathrm{q} \cdot \mathrm{min}} / \mathrm{a}$ & 0.41 & 0.53 & 0.45 & 0.63 \\
\hline $\mathrm{I}_{\mathrm{p}}$ & $1.6 \mathrm{MA}$ & $1.6 \mathrm{MA}$ & $1.5 \mathrm{MA}$ & $2.17 \mathrm{MA}$ \\
\hline$\beta$ & $0.53 \%$ & $0.68 \%$ & $0.68 \%$ & $0.68 \%$ \\
\hline$\beta_{\mathrm{p}}$ & 1.3 & 1.56 & 1.15 & 0.92 \\
\hline$\beta_{\mathrm{n}}$ & 1.4 & 1.7 & 1.45 & 1.45 \\
\hline
\end{tabular}

Recent advance in plasma profile measuring techniques, such as the Motional Stark Effect (MSE) q-profile and Electron Cyclotron Emission (ECE) electron temperature fluctuation diagnostics, have made it possible to study quantitatively MHD fluctuation behavior. One of interesting studies is the comparison of the experimentally observed mode structure with theoretical predictions. The mode structure is available as a radial displacement component, $\xi_{\text {r.exp }}(\mathrm{r})$, derived from the ECE radial $\mathrm{T}_{\mathrm{e}}(\mathrm{r})$ profile. Here, the spatial resolution of the fluctuation profile has been improved by combining profile data available from two ECE systems. The time evolution of $q(r, t)$-profile can be determined with the combination of MSE measurement and TRANSP [?] code.

In several aspects, the observed mode structure in ERS high $\beta$ disruptions is consistent with ideal MHD predictions. The detailed observations are summarized as follows:

- A global mode starts to grow as qmin approaches 2. The amplitude of the radial displacement profile, $\xi_{\text {r.exp }}(r)$, has a maximum value, $\xi_{\text {r.exp }}$, near $\mathrm{r} \simeq \mathrm{r}_{\mathrm{q} \cdot \min }$. 
- The amplitude of the radial displacement at the edge, $\xi_{\text {r.exp }}^{\text {edge }}$, estimated from Mirnov signal, is typically $1 / 7-1 / 4$ times $\xi_{\text {r.exp }}^{\max }$ which is expected from the ideal external kinks with wall boundary of $\mathrm{b} / \mathrm{a}=1.3-1.5$, where $\mathrm{b}$ is the wall minor radius.

- During the precursor period, both $\xi_{\text {r.exp }}^{\max }$ and $\xi_{\text {r.exp }}^{\text {edge }}$ increase in time with approximately the same growth rate until the thermal collapse takes place. This indicates that the mode structure, $\xi_{\text {r.exp }}(r)$, grows uniformly as a global mode.

- The observed mode frequency, $\omega_{\text {obs }} / 2 \pi$, seems not to propagate with the plasma rotation referred from the Doppler shift of impurity CVI charge recombination light. This relationship is different from the one expected for the resistive tearing mode, which propagates with the plasma rotation due to the tight coupling of the magnetic islands with the bulk plasma rotation. This mode slippage relative to the bulk plasma rotation is consistent with the ideal MHD hypothesis. The observed frequency can be explained by including kinetic effects, although the mode frequency is not far from the bulk plasma rotation frequency estimated with a neoclassical transport hypothesis.

In section 2 , the scheme for the determination of $\xi_{\text {r.exp }}$ will be discussed. The observed mode structures are presented in section 3 , and the comparison of the observed $\xi_{\text {r.exp }}$ with the theoretical prediction $\xi_{\text {r.the }}$ is given in the section 4. In section 5, the experimental results are summarized and comments are made on the ideal MHD theory based on the comparison of experiment and theory.

\section{Radial Displacement $\xi_{\text {rexp }}(\mathrm{r})$ Determination of Dis- ruption Precursors with Two ECE systems}

The observed ECE radiation frequency and its intensity are directly related to the plasma major radial location and the local electron temperature when the plasma density is high enough to satisfy the black body radiation criterion [22]. The ECE system is an excellent diagnostic for obtaining the local $\mathrm{T}_{\mathrm{e}}(\mathrm{r})$ profile and local fluctuations with a high time resolution $(\simeq 2 \mu \mathrm{sec})$. In TFTR, two grating polychromator systems for ECE referred as GPC1, GPC2 are operational with a toroidal separation of $126^{\circ}$. Both of them have 20 channels covering $100-120 \mathrm{~cm}$ in the major radial direction with channel separation $\simeq 5 \mathrm{~cm}$ compared to the $80-90 \mathrm{~cm}$ minor radius [22]. One issue for the quantitative study is the spatial resolution of fluctuation profiles. The resolution of $\simeq 5 \mathrm{~cm}$ by an ECE system is often too coarse for the ERS plasmas where 
the temperature gradient length is $\simeq 10-30 \mathrm{~cm}$. Furthermore, for the purely growing $\omega \simeq 0$ mode, monotonic evolution of a signal in time is hard to be recognized as an identifiable mode.

Here, it is shown that it is possible to improve the spatial resolution by combining the two profiles obtained from GPC1 and GPC2. The radial location of GPC1 and GPC2 can be independently moved so that the number of observation points can be increased. The difficulty of relating two ECE profiles together is due to the uncertainties of the relative radial location and the relative gain. Both of the systems are also vulnerable to calibration errors related to electronic circuitry drift.

The technique used here is a common mesh approach to minimize these uncertainties related to calibration factors. All channels of GPC1 signal are simultaneously analyzed with Michelson interferometer to determine independently the temperature profile [22]. Thus, the temperature profile of GPC1 serves as the base profile for this analysis. The other unit, GPC2, does not have the capability for independent calibration. In this process, the locations and the gain factors of $\mathrm{GPC} 2$ profile, $\mathrm{Y}_{\mathrm{GPC} 2}$, were re-normalized to minimize root-mean-square deviation compared with the GPC1 profile, $Y_{\mathrm{GPC} 1}$, during the MHD quiescent period by varying the relative gain of the channel, $\delta G_{j}$ and the radius correction, $\delta r_{j}$ for all GPC2 channels $(j=1-20)$ namely,

$$
\begin{gathered}
\delta \mathrm{W}_{\mathrm{j}}=\int\left[\mathrm{Y}_{\mathrm{GPC} 1}\left(\mathrm{r}_{\mathrm{j} . \mathrm{GPC} 1}, \mathrm{t}\right)-\left(1+\delta \mathrm{G}_{\mathrm{j}}\right) \mathrm{Y}_{\mathrm{GPC} 2}\left(\mathrm{r}_{\mathrm{j} . \mathrm{GPC} 2}+\delta \mathrm{r}_{\mathrm{j}}, \mathrm{t}\right)\right]^{2} \mathrm{dt} \\
\partial \delta \mathrm{W}_{\mathrm{j}} / \partial \delta \mathrm{G}_{\mathrm{j}}=0 \\
\partial \delta \mathrm{W}_{\mathrm{j}} / \partial \delta \mathrm{r}_{\mathrm{j}}=0
\end{gathered}
$$

where the time interval used is normally $1.5-2.5 \mathrm{sec}$ of MHD quiescent period. Typical values for these correction factors are $\delta r_{j}^{\prime} s \leq 1-2 \mathrm{~cm}$, and $\delta G_{j}^{\prime} s \leq$ $2-5 \%$. The common mesh radius, $\mathrm{r}_{\mathrm{c}, \mathrm{j}}$, is determined as the results of this minimization process. Although these values are relatively small, correction factors of $1-2 \mathrm{~cm}$ and $5 \%$ are important for the fluctuation levels which are $\leq 2-5 \%$ of the average profiles. With this process, the spatial resolution can be increased by a factor up to 1.2 - 2 depending upon the initial alignment of two systems. This also reduces systematic errors such as due to the hysteresis of the mechanical displacement of the grating plates when the plates are shifted to accommodate the various toroidal fields.

With this common mesh, not only oscillatory modes, but also even the purely growing $\omega \simeq 0$ mode can be analyzed with better accuracy. Assuming that the magnetic surface is a closed surface without a magnetic island (or outside the island if it does exist), the mode radial displacement profile $\xi_{\text {r.exp }}(\mathrm{r}, \mathrm{t})$, is calculated from 


$$
\xi_{\text {r.exp }}(\mathrm{r}, \mathrm{t}) \equiv \Delta \mathrm{T}_{\mathrm{e}} /\left(\partial \mathrm{T}_{\mathrm{e}} / \partial \mathrm{r}\right)
$$

The $\Delta T_{e}$ is defined;

(1) for oscillatory modes

$$
\Delta \mathrm{T}_{\mathrm{e}}=\left(\mathrm{T}_{\mathrm{e} . \mathrm{GPC} 1}\left(\mathrm{r}_{\mathrm{c} . \mathrm{j}}, \omega\right)+\mathrm{T}_{\mathrm{e} . \mathrm{GPC} 2}\left(\mathrm{r}_{\mathrm{c} . \mathrm{j}}, \omega\right)\right) / 2 .
$$

where $T_{\text {e.GPC1 }}\left(r_{c . j}, \omega\right)$ and $T_{\text {e.GPC } 2}\left(r_{c . j}, \omega\right)$ are the coefficients of the dominant mode frequency determined by Fourier decomposition and

(2) for the amplitude of the quasi-stationary purely growing $\omega \simeq 0$ modes

$$
\Delta \mathrm{T}_{\mathrm{e}}=\mathrm{T}_{\mathrm{e}}\left(\mathrm{r}_{\mathrm{c} . \mathrm{j}}, \mathrm{t}\right)-\mathrm{T}_{\mathrm{e}}\left(\mathrm{r}_{\mathrm{c} . \mathrm{j}}, \mathrm{t}_{\mathrm{ref}}\right)
$$

where the time evolution of the average profile is compared with the profile at a reference time, $t_{\text {ref }}$, immediately before the MHD mode onset on the common mesh radius, $r_{c}$.

The average of the profiles from GPC1 and GPC2 is useful for evaluating $\partial \mathrm{T}_{\mathrm{e}} / \partial \mathrm{r}$ and consequently minimizing uncertainty of the $\xi_{\mathrm{r} . \exp }(\mathrm{r})$ profile.

The Mirnov signals, measured at $\simeq 30$ toroidal and poloidal locations, were used to estimate the edge displacement value, $\xi_{\text {r.exp }}^{\text {edge }}$, by extrapolating from the Mirnov coil location to the plasma surface with the dependence factor $\left(b_{\text {Mirnov }} / a\right)^{m}$, where $b_{\text {Mirnov }}$ is the minor radial location of Mirnov coils and a is the plasma minor radius. Since the TFTR plasma is a circular configuration and the Mirnov coils are located close to the plasma surface, this approximation should be adequate. Typical values used are $\mathrm{b}_{\text {Mirnov }} / \mathrm{a}=1.2-1.3, \mathrm{~m}=5$ -6 , whenever the determination of $n, m$ values is possible. The uncertainty in the determination of $m$ value is a dominant factor in the uncertainties shown in the following figures.

The mode frequency is a useful parameter to characterize the cause of the observed mode. If the mode is related to the resistive tearing mode, it is expected to propagate with the bulk plasma rotation frequency since the magnetic islands is tightly coupled to the bulk plasma. For ideal modes, there have been several discussions of the ideal MHD mode frequency relative to the plasma rotation [23, 24]. The rotation frequency of bulk ions was estimated with neoclassical theory using TRANSP [21]. The direction of mode rotation is cited relative to the plasma current direction and the polarity is assigned in the right-handed cylindrical coordinate $(r, \phi, z)$.

\section{Observation of Mode Structure}

Here, we present typical examples of the mode structure for major disruption precursors determined with the process discussed above. The summary of the observations is listed in Table-II. 
The $q$-profiles used in the present analysis were deduced by using MSE $q-$ profiles available before the disruption events and by extrapolating close to the event time period with the TRANSP code. For example, in the $₫ 84011$ analysis, the MSE data was available up to $2.46 \mathrm{sec}$ and for the shot $\sharp 93260$ MSE data was up to $2.0 \mathrm{sec}$. For the shot $\sharp 91254$ without MSE measurement, the q-profile was prepared by TRANSP assuming the transport properties are similar to the plasma conditions which were documented by MSE measurement.

\section{Table II}

\begin{tabular}{|c|c|c|c|c|}
\hline & small & small & small & large \\
\hline & $\mathrm{r}_{\mathrm{q} \cdot \min }$ & $r_{q \cdot \min }$ & $r_{q \cdot \min }$ & $\mathrm{r}_{\mathrm{q} \cdot \min }$ \\
\hline shot & $\sharp 84011$ & $\sharp 84011$ & $\sharp 91254$ & $\sharp 93260$ \\
\hline time & $2.8 \mathrm{sec}$ & $3.08 \mathrm{sec}$ & $3.134 \mathrm{sec}$ & $2.497 \mathrm{sec}$ \\
\hline$\tau_{\text {growth }}$ & steady state & $\simeq 150 \mu \mathrm{sec}$ & $\simeq 120 \mu \mathrm{sec}$ & $\simeq 150 \mu \mathrm{sec}$ \\
\hline$r_{q \cdot \min } / \mathrm{a}$ & 0.41 & 0.53 & 0.45 & 0.63 \\
\hline$\xi_{r . e x p}^{\max }$ & $7 \mathrm{~mm}$ & $30 \mathrm{~mm}$ & $35 \mathrm{~mm}$ & $20 \mathrm{~mm}$ \\
\hline$\xi_{\text {r.exp }}^{e d g e}$ & $1 \mathrm{~mm}$ & $7 \mathrm{~mm}$ & $9 \mathrm{~mm}$ & $9 \mathrm{~mm}$ \\
\hline$\delta B$ & 0.5 gauss & 3 gauss & 2 gauss & 5 gauss \\
\hline $\mathrm{m} / \mathrm{n}$ & $5 / 2$ & $2 /(-1)$ & $2 /(-1)$ & $2 / 1$ \\
\hline$\omega_{o b s} / 2 \pi$ & $11 \mathrm{kHz}$ & $7 \mathrm{kHz}$ & $7 \mathrm{kHz}$ & $\simeq 0$ \\
\hline
\end{tabular}

\subsection{Plasma with Small $\mathrm{r}_{\mathrm{q} \cdot \mathrm{min}}$}

Figure 1 shows the time evolution of a reversed shear discharge that disrupted at $\beta_{\mathrm{n}}=1.7, \beta=0.68 \%, \beta_{\mathrm{p}}=1.56$, with $\mathrm{I}_{\mathrm{p}}=1.6 \mathrm{MA}$ at $\mathrm{t}=3.08 \mathrm{sec}(\sharp 84011)$. Prior to the major disruption precursor, the location of $q_{\min }$ was at $\mathrm{r}_{\mathrm{q} \text {.min }} / \mathrm{a}=$ 0.41 around $2.8 \mathrm{sec}$. An MHD mode with a frequency around $11 \mathrm{kHz}$ started to grow during the NBI prelude heating period with $\beta=0.53 \%, \beta_{\mathrm{n}}=1.4$, and $\beta_{\mathrm{p}}=1.3$. The mode has $\mathrm{m} / \mathrm{n}=$ odd $/ 2$ (counter-direction) near $\mathrm{q}=5 / 2$, (indicating $\mathrm{m} / \mathrm{n}=5 / 2$ ), based on the GPC1 and GPC2 observation. The mode persisted in a quasi-saturated manner until the final thermal collapse at $t=3.084 \mathrm{sec}$, as seen in Fig.1(c).

As $q_{\text {min }}$ approached 2 with $\mathrm{r}_{\mathrm{q} \cdot \mathrm{min}} / \mathrm{a}=0.53$ at $\mathrm{t}=3.08 \mathrm{sec}$ (a few msec before the thermal collapse), an additional $n=-1$ (co-direction) mode with slightly lower frequency started to grow, coexisting with the $\mathrm{m} / \mathrm{n}=5 / 2$ tearing mode(Fig.1(d)). The growth rate is $100-120 \mu$ sec with $\mathrm{m} / \mathrm{n}=$ even $/-1$ near the $\mathrm{q}=2$ surface, indicating large $2 / 1$ component. It is to be noted that the 
propagation direction is opposite to the $\mathrm{m} / \mathrm{n}=5 / 2$ tearing mode, indicating a possibility that the mode could be detached from the plasma rotation.

Let us discuss the mode structure of the steady state MHD activity at $t$ $=2.8 \mathrm{sec}$ with $\mathrm{m} / \mathrm{n}=5 / 2$ for the comparison with the disruption precursors. Figure 2 shows the q-profile and the radial mode displacement, $\xi_{\text {r.exp }}(r)$, vs. major radius for the $5 / 2$ mode at $t=2.8$ sec. The sensitivity to the $\mathrm{T}_{\mathrm{e}}$ gradient is shown in Fig.2. The maximum deviation shown in Fig.2 is calculated using either GPC1 or GPC2 profile for $\mathrm{T}_{\mathrm{e}}(\mathrm{r}, \mathrm{t})$ gradient and the solid line is calculated with the averaged $\mathrm{T}_{e}(\mathrm{r}, \mathrm{t})$ profile. This demonstrates the improvement of averaging two profiles to reduce the $\mathrm{T}_{\mathrm{e}}(\mathrm{r}, \mathrm{t})$ profile uncertainty. The radial displacement profile, $\xi_{\text {r.exp }}(\mathrm{r})$, has a broad maximum, $\xi_{\text {r.exp }}^{\max } \simeq 7 \mathrm{~mm}$, near the outer $q=5 / 2$ location $(\mathrm{R} \simeq 315 \mathrm{~cm}$ ) and decreases rapidly both toward outboard and inboard sides. The integrated Mirnov fluctuation level was $\delta \mathrm{B} \simeq 0.5$ Gauss, indicating a surface displacement value $\xi_{\text {r.exp }}^{\text {edge }} \simeq 0.7-1 \mathrm{~mm}$. The comparison of phase difference between the GPC1 and GPC2 signals indicates that the $5 / 2$ mode has a magnetic island structure. The $\xi_{\text {r.exp }}^{\max }$ is located only near the outboard $q=5 / 2$ region, not near the inboard $q=5 / 2$ region, suggesting that this $5 / 2$ tearing mode does not seem to be the resistive-g mode [12]. The relation to the plasma rotation frequency measured by the CVI emission is shown in Fig.2(c), suggesting that the mode propagates with the plasma rotation (the counter-direction) consistent with being a tearing mode. Details of the mode rotation will be discussed in section 4 .

At $t=3.08 \mathrm{sec}$, with $\mathrm{r}_{\mathrm{q} \cdot \mathrm{min}} / \mathrm{a}=0.53$, just before the thermal collapse, another slowly rotating mode, $\omega_{\text {obs }} / 2 \pi \simeq 7 \mathrm{kHz}$, was excited in addition to the already existing $\omega_{\text {obs }} / 2 \pi \simeq 10 \mathrm{kHz}$, as shown in Fig.3. The displacement profile, $\xi_{\text {r.exp }}(\mathrm{r})$, has a maximum slightly inside $\mathrm{r}_{\mathrm{q} \cdot \min }$ (and also near the $\mathrm{q}=2$ surface) and decreases gradually toward the edge. $\xi_{\text {r.exp }}^{\max }$ is $\simeq 3 \mathrm{~cm}$ and the edge displacement component, $\xi_{\text {r.exp }}^{\text {edge }} \simeq 7 \mathrm{~mm}$, estimated from the integrated Mirnov signal of $\delta \mathrm{B}=3$ Gauss. The ratio of $\xi_{\text {r.ex }}^{\max }$ to the $\xi_{\text {r.exp }}^{\text {edge }}$ is higher compared with the value from the resistive tearing $\mathrm{m} / \mathrm{n}=5 / 2$ mode at $\mathrm{t}=2.8 \mathrm{sec}$, and the spatial decay length of $\xi_{\text {r.exp }}(r)$ toward $r \geq r_{\text {q.min }}$ is more gradual than the one with the tearing $\mathrm{m} / \mathrm{n}=5 / 2$ mode. The mode seems to propagate in the co-direction $(n=-1)$, opposite to the observed rotation frequency $\omega_{0}$. That means the $n=-1$ mode rotation seems to be free from the plasma rotation. This is in contrast to the behavior of the resistive tearing mode as shown with $5 / 2$ modes.

In Fig.4, we present the observations on another discharge $(\sharp 91254)$ which also has a small $\mathrm{r}_{\mathrm{q} \cdot \min }\left(\mathrm{r}_{\mathrm{q} \cdot \min } / \mathrm{a}=0.45\right)$. In this discharge, the plasma parameters at the thermal collapse are $\beta=0.68 \%, \beta_{\mathrm{n}}=1.45, \beta_{\mathrm{p}}=1.15$, and qedge $=6.2$ with $\mathrm{I}_{\mathrm{p}}=1.50 \mathrm{MA}$. As shown in Fig.4 (a), the numerically integrated Mirnov signal and $\xi_{\text {r.exp }}$ show a similar growth rate with a time constant of $\simeq 120 \mu \mathrm{sec}$, until the thermal collapse. The value of $\xi_{\mathrm{r} . \max }$ reached $2-3 \mathrm{~cm}$ 
before the thermal collapse, and $\delta \mathrm{B}$ reached up to 2 Gauss. The value of $q_{\text {min }}$ reached 2 within the accuracy of the MSE-q measurement (Fig.4(b)). The mode has two dominant frequencies, $\omega_{\text {obs }} / 2 \pi$, one at $\simeq 7 \mathrm{kHz}$ and the other $\simeq 21 \mathrm{kHz}$, as seen on the trace of the displacement maximum $\xi_{\text {r.exp }}$ in Fig.4(c). Based on the phase between the GPC1 and GPC2 signals, the mode component is $\mathrm{m} / \mathrm{n}=$ even $/-1$ near the $\mathrm{r}_{\mathrm{q} \text { min }}$ area. On the plasma surface, the mode is $\mathrm{m} / \mathrm{n}=6 /-1$ for $7 \mathrm{kHz}$. For the higher frequency $21 \mathrm{kHz}$ activity, the mode is likely to be $\mathrm{n}=-3$, although the mode identification was impossible due to higher $\mathrm{m} / \mathrm{n}$ values. At the radial location of $\xi_{\text {r.exp }}^{\max }$, the observed frequency, $\omega_{\text {obs }} / 2 \pi$, is $\geq+7 \mathrm{kHz}$ and is in the opposite direction to the observed rotation frequency, $\omega_{0} \simeq-(1-2) \mathrm{kHz}$ in the co-direction near $\mathrm{r}=\mathrm{r}_{\mathrm{q} \text {.min }}$ indicating that the mode slips with respect to the plasma rotation. This relationship of the mode propagation direction relative to the plasma rotation frequency is similar to the case of $\sharp 84011$. The mode structures for $7 \mathrm{kHz}$ and $21 \mathrm{kHz}$ are shown in Fig.4(d). The Fourier decomposed amplitude for $21 \mathrm{kHz}$ is $1 / 4$ of the $7 \mathrm{kHz}$ mode amplitude. Although the amplitudes differ from each other, the $\xi_{\text {r.exp }}^{\max }$ location and the mode radial spreading are similar at both frequencies, indicating that the higher frequency component may be coupled to the lower frequency structure rather than an independent $n=-3$ ideal kink mode. The $\xi_{\text {r.exp }}(\mathrm{r})$ profile with $\mathrm{r}_{\mathrm{q} \text {.min }} / \mathrm{a}=0.45$ is narrower than the $\mathrm{r}_{\mathrm{q} \text {.min }} / \mathrm{a}=0.53$ $(\sharp 84011)$, indicating that the spreading of $\xi_{\text {r.exp }}(r)$ depends on the location of $\mathrm{r}_{\mathrm{q} \cdot \min }$.

\subsection{Plasmas with Large $\mathrm{r}_{\mathrm{q} . \mathrm{min}}$}

Figure 5 shows a case of larger $\mathrm{r}_{\mathrm{q} \text { min }} / \mathrm{a}=0.63$ with $\beta=0.68 \%, \beta_{\mathrm{n}}=1.45, \beta_{\mathrm{p}}=$ $0.92, q_{\text {edge }}=4.4$ and $\mathrm{I}_{\mathrm{p}}=2.17 \mathrm{MA}(\sharp 93260)$. The mode is nearly toroidally stationary and purely growing, with the real frequency $\omega_{\text {obs }} / 2 \pi \simeq 0$. The mode structure was calculated with Eq.6 for $\omega \simeq 0$ mode. As shown in Fig.5(a,b), the $\xi_{\text {r.exp }}^{\max }$ and $\delta \mathrm{B}$ increased monotonically in time with approximately the same time constant of $120-150 \mu \mathrm{sec}$ until the thermal collapse was initiated. Figures $5(\mathrm{c}, \mathrm{d})$ show that in the large $\mathrm{r}_{\mathrm{q} . \mathrm{min}} / \mathrm{a} \simeq 0.63$, the displacement profile, $\xi_{\text {r.exp }}(\mathrm{r})$, is much broader and the edge displacement, $\xi_{\text {r.exp }}^{\text {edge }}$, is larger compared with the one associated with $\mathrm{r}_{\mathrm{q} \cdot \mathrm{min}} / \mathrm{a}=0.45(\sharp 91254)$ and $=0.53(\sharp 84011)$. Both of the observed plasma rotation frequency and the observed rotation frequency are nearly zero within experimental errors so that it is hard to relate the mode rotation to the plasma rotation frequency. 


\section{Comparison of Radial Displacement, $\xi_{\text {r.exp }}(\mathrm{r})$, with ideal MHD theory}

Theoretical stability analysis is based on a reconstruction of the equilibrium using the plasma pressure- and q-profiles from the TRANSP code. The PEST [25] code is then used to determine the stability properties. These plasmas are found to be unstable to an $n=1$ free boundary external kink mode. In this context, we address the role of the vacuum vessel and hardware in the external kink mode.

\subsection{Predictions by Ideal MHD Theory}

Figure 6(a) shows the PEST code prediction for the poloidally decomposed component $\xi_{\text {the.m }}(\mathrm{r})$ of an unstable mode with b/a $=1.5(\sharp 93260)$. The $\mathrm{m}=2$ component, $\xi_{\text {r.the.m=2 }}(\mathrm{r})$, is internally driven and reaches a maximum near $\mathrm{r} / \mathrm{a}$ $=0.4$, close to the location of zero magnetic shear. The additional contribution to the instability, externally driven, is due to the current gradient near the surface, producing a large $\xi_{\text {r.the.m=5 }}(\mathrm{r})$ component. Other m-components, $\mathrm{m}$ $=3,4$, provide the means for poloidal coupling. The expected displacement, $\xi_{\text {r.the }}(\mathrm{r})$, was calculated by summing the strength of $\sum_{m} \xi_{\text {r.the.m }}(r)$ over all mcomponents on the $z=0$ mid-plane. This value should be equivalent to the experimental value, $\xi_{\text {r.exp }}(\mathrm{r})$.

An important feature of the mode structure is the ratio of the mode amplitude at the edge and at the maximum, $\xi_{\text {r.the }}^{\max } / \xi_{\text {r.the }}^{\text {edge }}$. This ratio and the normalized growth rate, $\left(\gamma \tau_{\mathrm{A}}\right)^{2}$, are shown with varying b/a in Fig.6(b) for the shot $(\sharp 93260)$, where $\tau_{A}$ is the poloidal alfven transit time. As $\mathrm{b} / \mathrm{a}=1.3$ is approached, the growth rate, $\left(\gamma \tau_{\mathrm{A}}\right)^{2}$, is reduced, indicating that the mode has a finite edge driven component. The marginal wall location, $\mathrm{b}_{\mathrm{TFTR}} / \mathrm{a}$, for this configuration seems to be $\simeq 1.3$. On the other hand, the increase of the ratio of $\xi_{\mathrm{r} \text {.the }}^{\max }$ to the $\xi_{\text {r.the }}^{\text {edge }}$ with lower $\mathrm{b} / \mathrm{a}$ suggests that the mode is also driven by the internal region presumably in the $q^{\prime} \simeq 0$ domain. For $b / a \simeq 2$, the internally driven and externally driven components are comparable. These results as shown in Fig.6(a,b) indicate that the dominant contribution of the instability with $\mathrm{b} / \mathrm{a}=1.3-1.5$ is the internally driven $\mathrm{m}=2$ component and the high-m coupling broadens the mode structure toward the plasma edge. In another words, the source of edge driven kink plays a lesser role in the experimental condition.

The geometry of the TFTR vacuum vessel is complex with various flanges and carbon tiles. With additional consideration of the radius irregularity due to flange locations, it is reasonable to assume that the effective wall location is $\mathrm{b}_{\text {TFTR }} / \mathrm{a}=1.3$ for the theoretical analysis. In the following, the value of $\mathrm{b} / \mathrm{a}=1.5$, slightly higher than $\mathrm{b}_{\text {TFTR }} / \mathrm{a}=1.3$, is used in the calculation in order 
to avoid the complexity of the actual detailed hardware arrangement.

Another important aspect of the results is that the theoretical normalized $\beta$ limit for larger $r_{\mathbf{q} \text { min }}$ is much smaller than for smaller $r_{\mathbf{q} \text { min }}$ case. For the small $\mathrm{r}_{\mathrm{q} \cdot \mathrm{min}}$ case, $\left(\mathrm{r}_{\mathrm{q} \cdot \mathrm{min}} / \mathrm{a}=0.53(\sharp 84011)\right)$, the calculated marginal onset of normalized beta was found to be $\beta_{\mathrm{n} \text {.the }}=1.7$. For the large $\mathrm{r}_{\mathrm{q} \cdot \min },\left(\left(\mathrm{r}_{\mathrm{q} \cdot \mathrm{min}} / \mathrm{a}=\right.\right.$ 0.63 case $(\sharp 93260))$, the marginal condition was $\beta_{\mathrm{n} \text {.the }}=1.3$. These values are in good agreement with the experimentally achieved value of $\beta_{\text {n.exp }}=1.7$ for $\mathrm{r}_{\mathrm{q} \cdot \min }=0.53$ and $\beta_{\mathrm{n} \cdot \exp }=1.45$ for $\mathrm{r}_{\mathrm{q} \cdot \min }=0.65$ respectively. According to the current profile analysis, lower $\beta$ prediction seems to be related to the larger edge current gradient for larger $r_{q \cdot m i n}$ configuration. The experimental difficulty of producing the larger $\mathrm{r}_{\mathrm{q} \text {.min }}$ configuration with larger plasma current may be related to lower $\beta_{n}$ limit.

\subsection{Comparison of $\xi_{\mathrm{r}}$}

Figure $7(\mathrm{a})$ shows the comparison of the experimental radial displacement profile, $\xi_{\text {r.exp }}(\mathrm{r})$ with the theoretical prediction, $\xi_{\text {r.the }}(\mathrm{r})$ for smaller $\mathrm{r}_{\text {q.min }} / \mathrm{a}=$ $0.53(\sharp 84011)$. The maximum $\xi_{\text {r.the }}(\mathrm{r})$ was normalized to the observed $\xi_{\text {r.exp }}$. The observed $\xi_{\text {r.exp }}(\mathrm{r})$ profile, including the edge value of $\xi_{\text {r.exp }}^{\text {edge }}$ estimated from the Mirnov signal, is in good agreement with $\xi_{\text {r.the }}(\mathrm{r})$. Figure 8(a) shows the comparison of the mode structure for the larger $\mathrm{r}_{\mathrm{q} . \mathrm{min}} / \mathrm{a}=0.63$ with higher plasma current $\mathrm{I}_{\mathrm{p}}=2.2 \mathrm{MA}(\sharp 93260)$. The overall behavior of the observed amplitude, $\xi_{\text {r.exp }}(\mathrm{r})$, also agrees with $\xi_{\text {r.the }}(\mathrm{r})$.

It is interesting to note that when the $\mathrm{r}_{\mathrm{q} \text {.min }} / \mathrm{a}$ was shifted outward to 0.45 $(\sharp 91254) 0.53(\sharp 84011)$ and $0.63(\sharp 93260)$, the structure became wider, as seen in Figs. 3, 4 and 5. This is also consistent with the predictions of ideal MHD theory. So far, the predicted and observed mode structures have been compared at a given time. A question arises as to whether the mode pattern is sustained while the mode grows. Figure 9 shows the relation between $\xi_{\text {r.exp }}^{\text {edge }}(t)$ and $\xi_{\text {r.exp }}^{\max }(\mathrm{t})$ during the precursor time until the thermal collapse. The modes shown here are purely growing $\omega \simeq 0$ modes. The mode became detectable at the $1 \mathrm{~mm}$ level of $\xi_{\text {r.exp }}^{\max }(\mathrm{r})$ and $\simeq 0.5 \mathrm{~mm}$ level of the $\xi_{\text {r.exp }}^{\text {edge }}(\mathrm{t})$ with a 0.2 Gauss magnetic perturbation on the Mirnov coils. The precursors maintained approximately the same ratio of $\xi_{\text {r.exp }}^{\max }(\mathrm{t})$ to $\xi_{\text {r.exp }}^{\text {edge }}(\mathrm{t})$ as they grew, indicating that these modes may have sustained its global structure intact and other modes may not have been excited until the collapse occurs, once the internal amplitude reaches finite $\approx 1 \mathrm{~cm}$.

\subsection{Mode Frequency}

As shown in Fig.1, two modes coexisted simultaneously. The mode bound to the plasma rotation is likely to be a resistive tearing mode. The direction of 
the other mode seems free from the plasma rotation (at least from the impurity ions). Here let us discuss possible causes of the apparent frequency difference; one is the kinetic effect and the other is the bulk plasma rotation frequency which may differ from the CVI impurity rotation frequency.

In the following we include the kinetic effect on the ideal kink mode. With the framework of Ref. [23], the ideal MHD mode slips from the bulk plasma rotation frequency with the effect of ion diamagnetic frequency, $\omega_{i}^{*}$. At the maximum expected growth rate, the mode frequency may shift by $\Delta \omega \simeq 1 / 4-1 / 2$ times $\omega_{i}^{*}$. The estimate was calculated with the profile parameters obtained through TRANSP analysis. Figure $7(\mathrm{~b})$ shows the possible range of the frequency estimated for the case of small $\mathrm{r}_{\mathrm{q} \cdot \min } / \mathrm{a}=0.53$. The observed frequency is in agreement with the frequency slipping by $1 / 2$ times $\omega_{i}^{*}$ from the observed plasma rotation frequency. The possible $\omega_{i}^{*}$ effect for the large $r_{q \cdot \min } / \mathrm{a}=0.63$ case is shown in Fig.8(b). This data is also consistent with the frequency difference by $\leq 1 / 4$ times $\omega_{\mathrm{i}}^{*}$. The best match for the frequency observed $\omega_{\mathrm{obs}}$ is near $\simeq \Omega_{0}+\omega_{i}^{*} / 4$, as expected by the theory [23].

However, in the above discussion, the observed plasma rotation frequency is for the CVI impurity. Thus, the rotation for the bulk plasma ions may be different. The possible bulk rotation profiles for deuterium are calculated in a steady state equilibrium, assuming that the transport process is fully described by the neoclassical coefficients [27] and the measured CVI velocity. Since the transport properties in the ERS regime have the ion transport coefficient not far from neoclassical in the regime, $r \simeq \mathrm{r}_{\mathrm{q} \cdot \min }$, the estimate with neoclassical transport is expected to be a reasonable choice for ions. Not only the toroidal rotation provides the drift frequency, but also the poloidal rotation could also modify the observed frequency by $\mathrm{B}_{t} / \mathrm{B}_{\mathrm{p}}$ times the poloidal rotation frequency. The magnitude of this component was calculated to be comparable to the toroidal rotation. Recently, the measurement of the poloidal rotation velocity has been made and it has been reported that the poloidal rotation can be larger than the neoclassical values [28]. However, the present discussion is constructed using only the toroidal rotation to obtain qualitative behavior with neoclassical correction. As shown in Fig.7(c), the observed frequency $\left(\omega_{\text {obs }} / 2 \pi \simeq 7 \mathrm{kHz}\right.$ ) is not far from the bulk deuterium rotation velocity for the smaller $\mathrm{r}_{\mathrm{q} \text {.min }} / \mathrm{a}=0.53$. For the larger $\mathrm{r}_{\mathrm{q} . \mathrm{min}} / \mathrm{a}=0.63$ case shown in Fig.8(c), the observed frequency $\left(\omega_{\text {obs }} \simeq 0\right)$ is again consistent with the calculated bulk rotation frequency.

The experimental observation indicates that the global $n=-1$ mode slips from the plasma rotation. However, from these analyses, it is difficult to conclude definitely whether the magnitude of the slippage is consistent with the ideal MHD theory with kinetic effect, since the bulk plasma rotation is not clearly determined. For further clarification for kinetic effects, the understanding of the neoclassical process is the most important issue. 


\section{Discussion}

In summary, not only do the onset conditions of major disruption precursors, such as the $\beta$ and $\beta_{\mathrm{n}}$, agree with the predictions from ideal MHD theory, but the observed profiles of the mode structure are also consistent with theoretical predictions in the following aspects.

(1) The relationship between the $\mathrm{m}=2$ component at the inner plasma and the $\mathrm{m} \simeq 5-6$ at the edge agree with predictions shown in Fig.6.

(2) The broader amplitude with larger $r_{q \text { min }}$ is consistent with ideal MHD theory.

(3) The profile of $\xi_{\text {r.exp }}(\mathrm{r})$ is in agreement with the ideal MHD predictions as shown in Fig.7 and 8.

(4) The ratio of displacement at the center and at the edge is also consistent with the ideal MHD predictions (Fig.7 and 8).

(5) The amplitude strength both near the magnetic axis and the plasma edge increased monotonically in time, indicating that the mode can still be reasonably described by linear ideal MHD analysis.

(6) The mode rotation frequency is not locked to the plasma rotation frequency.

Thus, ideal MHD theory may serve as a guide to MHD activity analysis in the Enhanced Reversed Shear configuration. It should be noted, however, that the $\xi_{\text {r.the }}(\mathrm{r})$ was normalized to the $\xi_{\text {r.exp }}^{\max }$. Thus, the significance of the agreement of the displacement profile $\xi_{\mathrm{r}}(\mathrm{r})$ should be interpreted as consistency of $\xi_{\mathrm{r}}(\mathrm{r})$ for $\mathrm{r} \geq \mathrm{r}_{\mathrm{q} \text {.min }}$ between the ideal MHD theory prediction and the observed $\xi_{\mathrm{r}}$ behavior. Once $\xi_{\mathrm{r}}(\mathrm{r})$ at $\mathrm{r} \geq \mathrm{r}_{\mathrm{q} \text {.min }}$ is properly described by ideal MHD theory, ideal MHD theory should provide a guide to improving the domain $r \geq r_{q . m i n}$. For example, stronger mode coupling at $r \geq r_{q . m i n}$ should improve the achievable beta limit by making the wall stabilization more effective. Stronger mode coupling at $r \geq r_{q \text { min }}$ can be obtained by increasing the toroidicity by lowering the aspect ratio or by increasing the poloidal coupling with the stronger plasma shaping. The next crucial issue for verification of ideal MHD is whether the theory can suggest new stabilizing schemes.

Another issue is the relationship of the mode frequency to the plasma rotation frequency. The mode rotation seems to slip from the plasma rotation, although the experimentally available information is not sufficient to evaluate 
the bulk rotation frequency with neoclassical hypothesis. The further experimental verification of frequency difference between the mode and the plasma rotation is very important. These studies will provide an opportunity to clarify the role of the kinetic effects on the other ideal modes like $1 / 1$ internal modes.

The experimental results analyzed here are only for the precursor process. The final thermal crash process which triggers the disruption remains unan-

swered. While the localized measurements by ECE and MSE diagnostics have opened a new era in the analysis of the internal MHD structure as reported, better understanding of this final triggering process still requires further development of diagnostics such as 3-dimensional MHD diagnostics to understand the sudden ergodic thermal collapse.

\section{Acknowledgment}

The authors express their appreciation to the technical, engineering and scientific staff of TFTR group. The authors also thank Dr. P. Savrukhin for helpful discussions and comments. This work was supported by the US Department of Energy under contract No. DE-AC02-76-CH03037.

\section{References}

[1] Tuda T. et AK., in Plasma physics and Controlled Nuclear Fusion Research (Proc. Int. Conf. 1984) Vol.II, IAEA, Vienna (1984) 173

[2] Troyon F. et al., Plasma Phy. Controlled Fusion 26 (1984) 209

[3] Strait E. J. et al., Phys. Plasmas 1 (1994) 1415

[4] Taylor T. et al., in Plasma physics and Controlled Nuclear Fusion Research (Proc. Int. Conf.1992) Vol.I, IAEA, Vienna (1993) 167

[5] Ishida S. et al., in Plasma physics and Controlled Nuclear Fusion Research (Proc. Int. Conf.1992) Vol.I, IAEA, Vienna (1992) 219

[6] Okabayashi M. et al., in Plasma physics and Controlled Nuclear Fusion Research (Proc. Int. Conf. 1986) Vol.1, IAEA, Vienna (1987) 275

[7] Sabbagh S.A. et al., Phy. Plasmas B 3 (1991) 2277

[8] Bussac M. N. et al., Phys. Rev. Lett. 35 (1975) 1638

[9] Wesson J. A. et al, Plasma Phy. and Contr. Fusion, 28 (1986) 243.

[10] Migliuolo S. et al, Nuclear Fusion 33 (1993) 1721 
[11] Bell M. et al., Phys. Plasmas 4, (1997) 1714

[12] Manickam J. et al., in 16th Plasma physics and Controlled Nuclear Fusion Research, Vol. I, IAEA, Vienna(1997) 453

[13] Ishida S. et al., in 16th Plasma physics and Controlled Nuclear Fusion Research, Vol. I, IAEA, Vienna(1997) 315

[14] McGuire K. et al., in 16th Plasma physics and Controlled Nuclear Fusion Research, Vol. I, IAEA, Vienna(1997) 19

[15] Fredrickson E. et al., Bull. Am. Phy. Soc. 41 1527(1996)

[16] Lazarus E.A. et al., in 16th Plasma physics and Controlled Nuclear Fusion Research, Vol.I, IAEA, Vienna(1997) 199.

[17] McGuire K. M. et al., Plasma Phys. Controlled Nuclear Fusion vol 1, (1987) 421

[18] Fredrickson E. D. et al., Phys. Plasmas 2 (1995) 4216

[19] Park W. C. et al., Phys. Rev. Lett. 75 (1995) 1763

[20] Chang Z. et al., Phys. Rev. Lett. 74 (1995) 4663

[21] Goldston, R.J. et al., J. Comput. Phy. 43 (1981) 61

[22] Janos A. et al., Rev. Sci. Instrum. 66 (1995) 668

[23] Pearlstein. L. D and Freidberg J. P., Phys. Fluids 21 (1978) 1218

[24] Ara G. et al., Annals of Physics, 112 (1978) 443

[25] Grimm, R.C. et al., J. Comput. Phy. 49 (1982) 94

[26] Wesson J. A. et al., Nuclear Fusion 29 (1989) 641

[27] Houlberg, W. A. et al., Phys. Plasmas 4 (1997) 3230

[28] Bell R. et al., Bull. Am. Phys. Soc. 42 (1997) 1945 (also submitted to Plasma Phys. and Controlled Fusion) 


\section{Figure Captions}

Figure 1

Time behavior of plasma parameters and signals of MHD activity in an ERS discharges in TFTR

(a) The plasma current vs. time.

(b) The $4-5 \mathrm{MW}$ of NBI was applied to maintain the hollow profile from $t$ $=0.7$ sec. The main heating was started from 2.4 sec.

(c) The Mirnov oscillation, the $\mathrm{m} / \mathrm{n}=5 / 2$ mode started to build up after the main heating power was on at $2.6 \mathrm{sec}$ and continued up to the thermal collapse.

(d) The frequency spectrum of the Mirnov signal immediately before the thermal collapse. Near the major disruption, the $\mathrm{n}=-1$ mode (presumably $\mathrm{m} / \mathrm{n}=2 /-1$ mode) rapidly grows in addition to the saturated $\mathrm{m} / \mathrm{n}=$ $5 / 2$ tearing mode.

Figure 2

The displacement profile $\xi_{\text {r.exp }}(\mathrm{r})$ of the precursor and the rotation velocity of the shot $[\sharp 84011]$ at $t=2.8 \mathrm{sec}$

(a) MSE q-profile

(b) $\xi_{\text {r.exp }}(\mathrm{r})$

(c) rotation profile

Figure 3

The mode structure with $n=-1$ mode before the disruption at $t=3.08 \mathrm{sec}$ $[\sharp 84011]$

(a) MSE q-profile

(b) fluctuation mode structure $\xi_{\text {r.exp }}(\mathrm{r})$ and the edge displacement $\xi_{\text {r.exp }}^{\text {edge }}$ estimated from the Mirnov signal

(c) rotation frequency profile

Figure 4

The small $\mathrm{r}_{\text {q.min }} /$ a oscillatory case $[\sharp 91254]$. The amplitudes are shown for two frequency components appeared in the signals. 
(a) The Mirnov amplitude and displacement $\xi_{\text {r.exp }}$ vs. time

(b) MSE q profile

(c) radial displacement $\xi_{\text {r.exp }}(\mathrm{r})$

(d) plasma rotation profile

Figure 5

The case for large $r_{q . m i n} / a$ with the shot $[\sharp 93260]$

(a) Mirnov signal and displacement $\xi_{\text {r.exp }}^{\max }$

(b) MSE q-profile

(c) fluctuation mode structure and the displacement calculated from the Mirnov signal

(d) plasma rotation profile

Figure 6

The mode structure calculated with ideal MHD theory. The calculation was done with PEST using the plasma profiles given by TRANSP analysis

(a) displacement amplitude $\xi_{\text {r.exp }}(\mathrm{r})$

(b) effect of the wall location on the the ratio of $\xi_{\text {r.the }}^{\text {max }} / \xi_{\text {r.the }}^{\text {edge }}$

Figure 7

The comparison of the observed $\xi_{\text {r.exp }}(r)$ with $\xi_{\text {r.the }}(r)$ by PEST for the shot $[\sharp 84011]$ at $t=3.08 \mathrm{sec}$.

(a) mode amplitude

(b) the estimated $\omega_{i}^{*}$ effect on the ideal kink mode

(c) the estimated bulk plasma rotation frequency with neoclassical theory

Figure 8

The comparison of the observed $\xi_{\text {r.exp }}(r)$ with $\xi_{\text {r.the }}(r)$ predicted by PEST for the shot $[\sharp 93270]$

(a) mode amplitude 
(b) estimated $\omega_{i}^{*}$ effect on the ideal kink mode

(c) estimated bulk plasma rotation frequency with neoclassical theory

\section{Figure 9}

$\xi_{\text {r.exp }}^{\text {edge }}(t)$ vs. $\xi_{\text {r.exp }}^{\max }(t)$ for four shots approaching disruptions in ERS phase. 


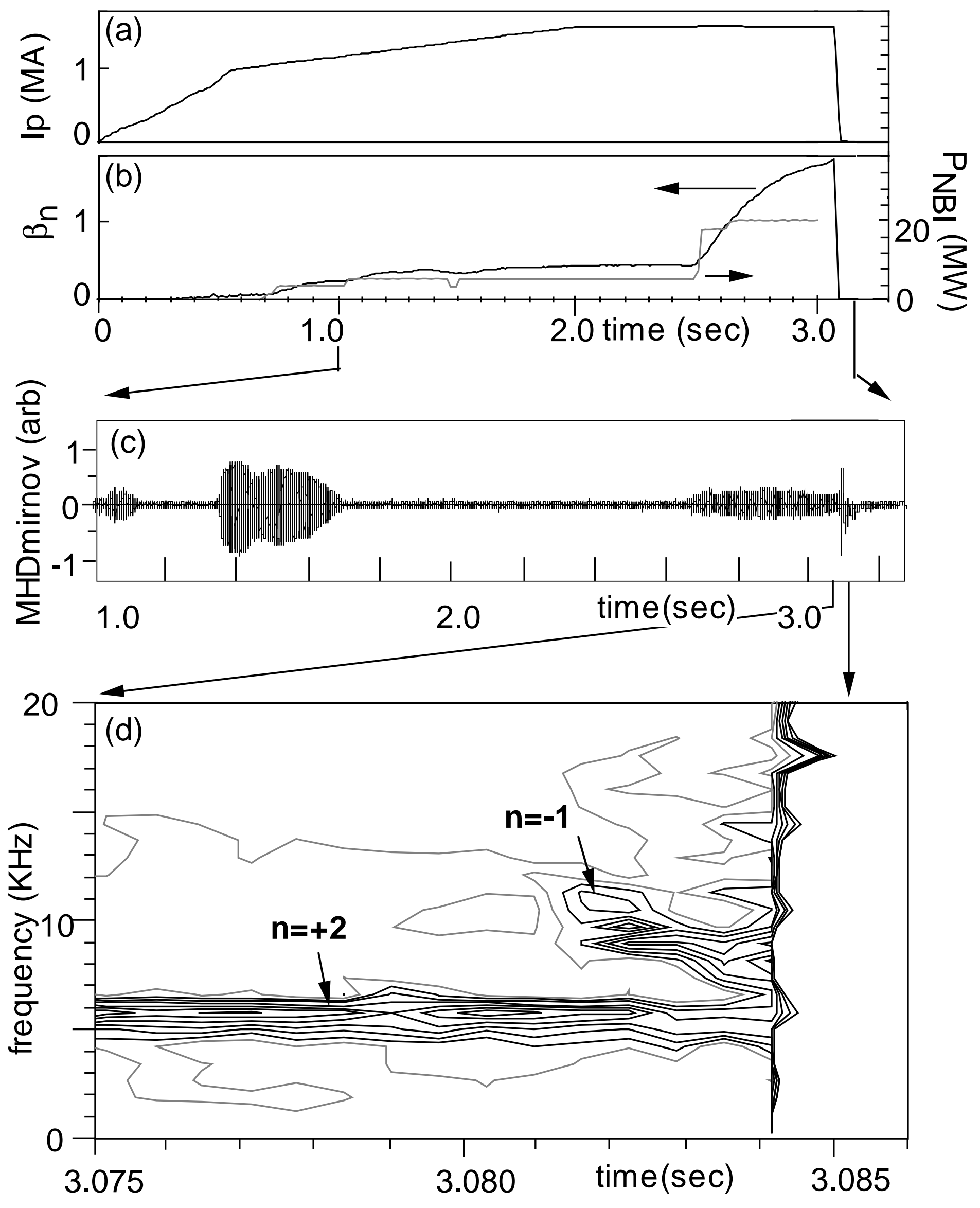




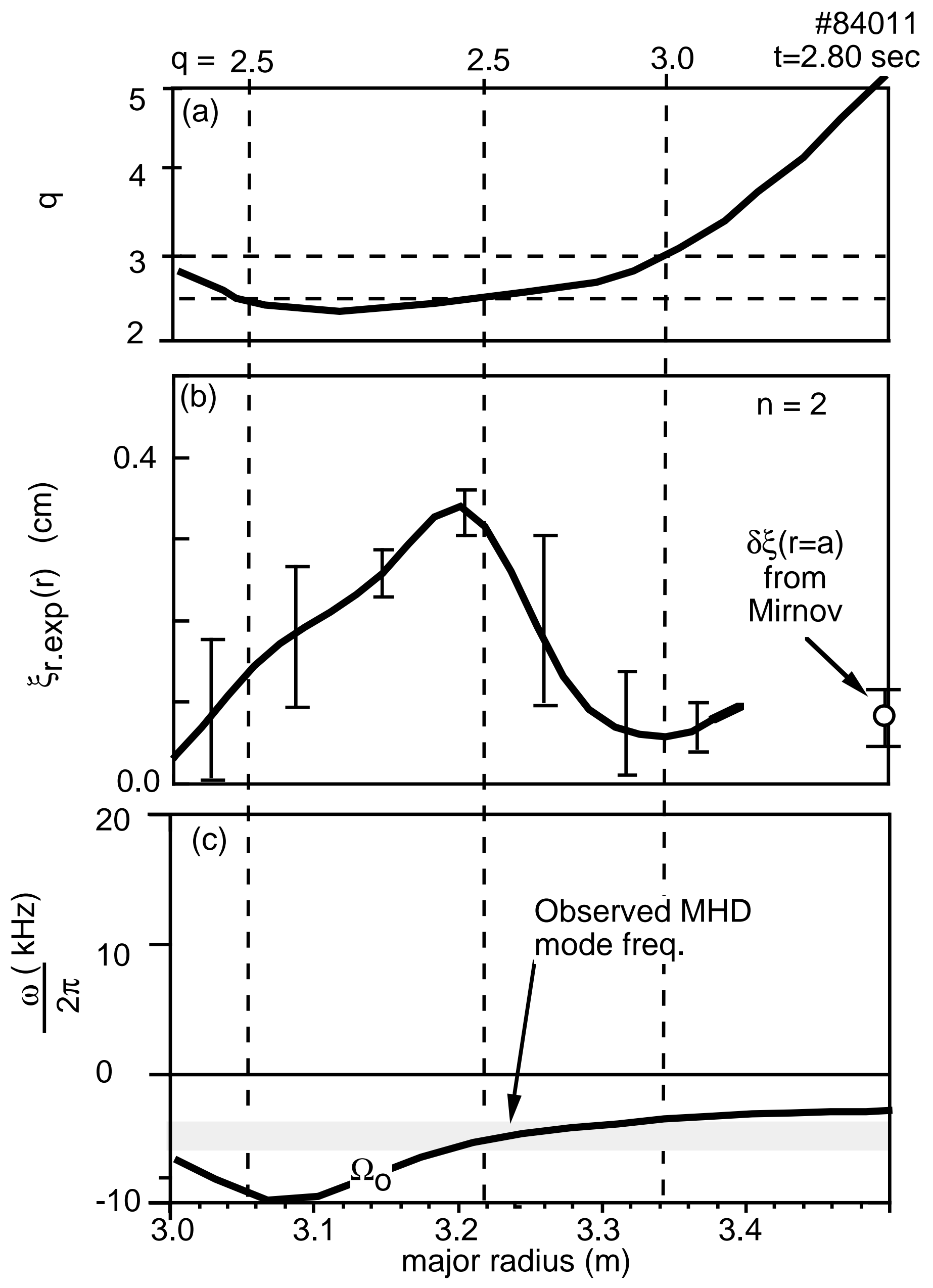



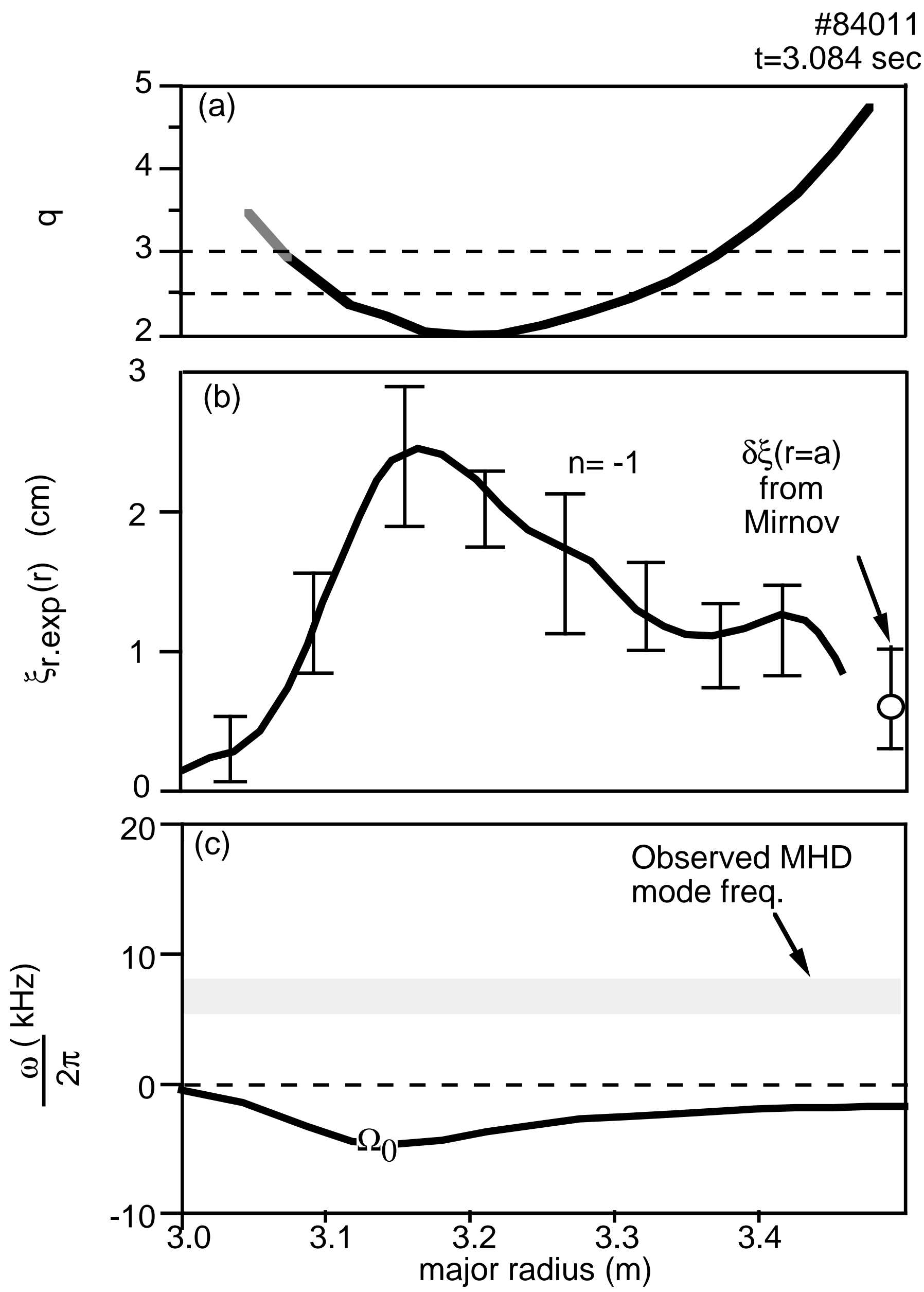

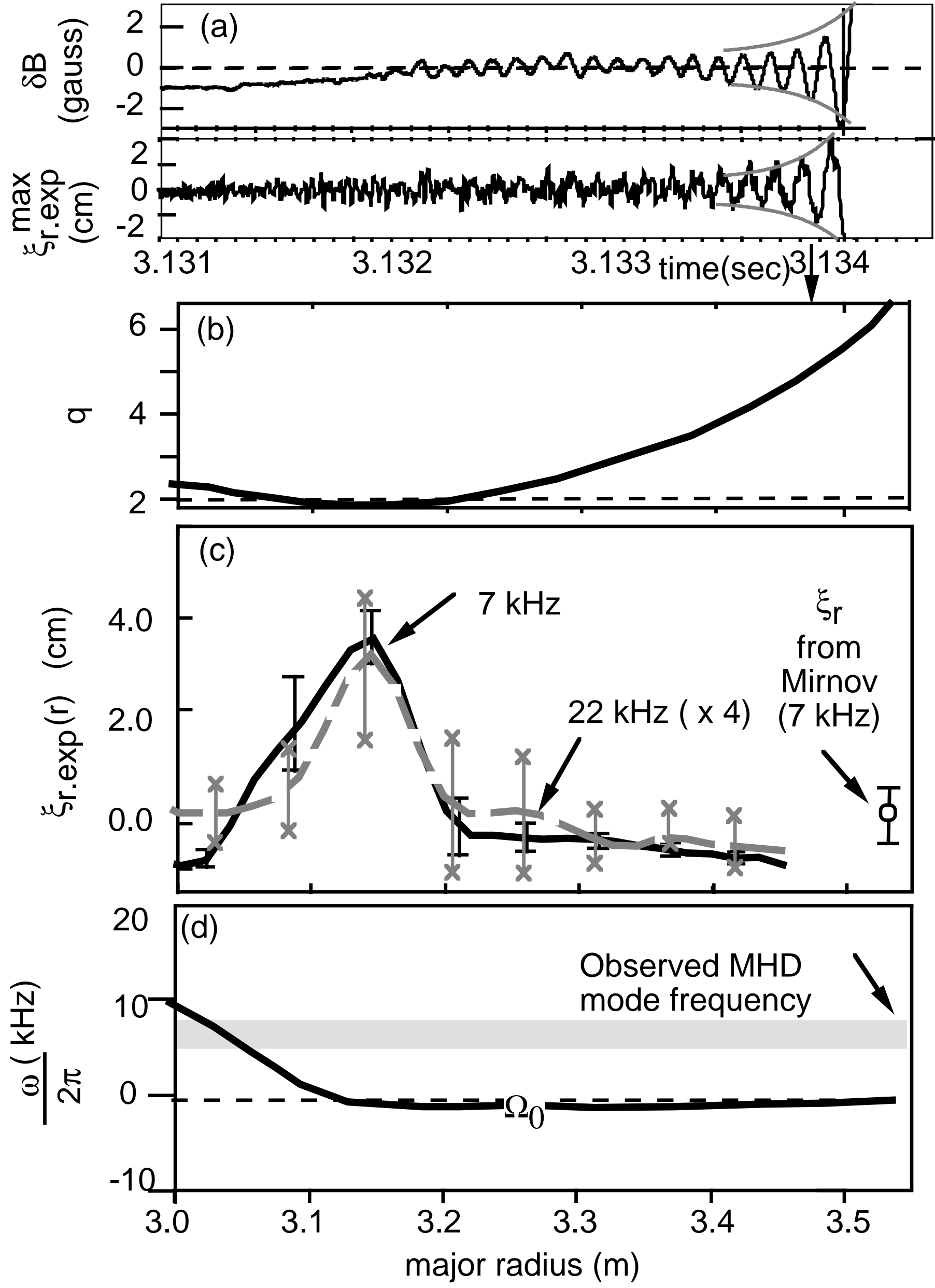

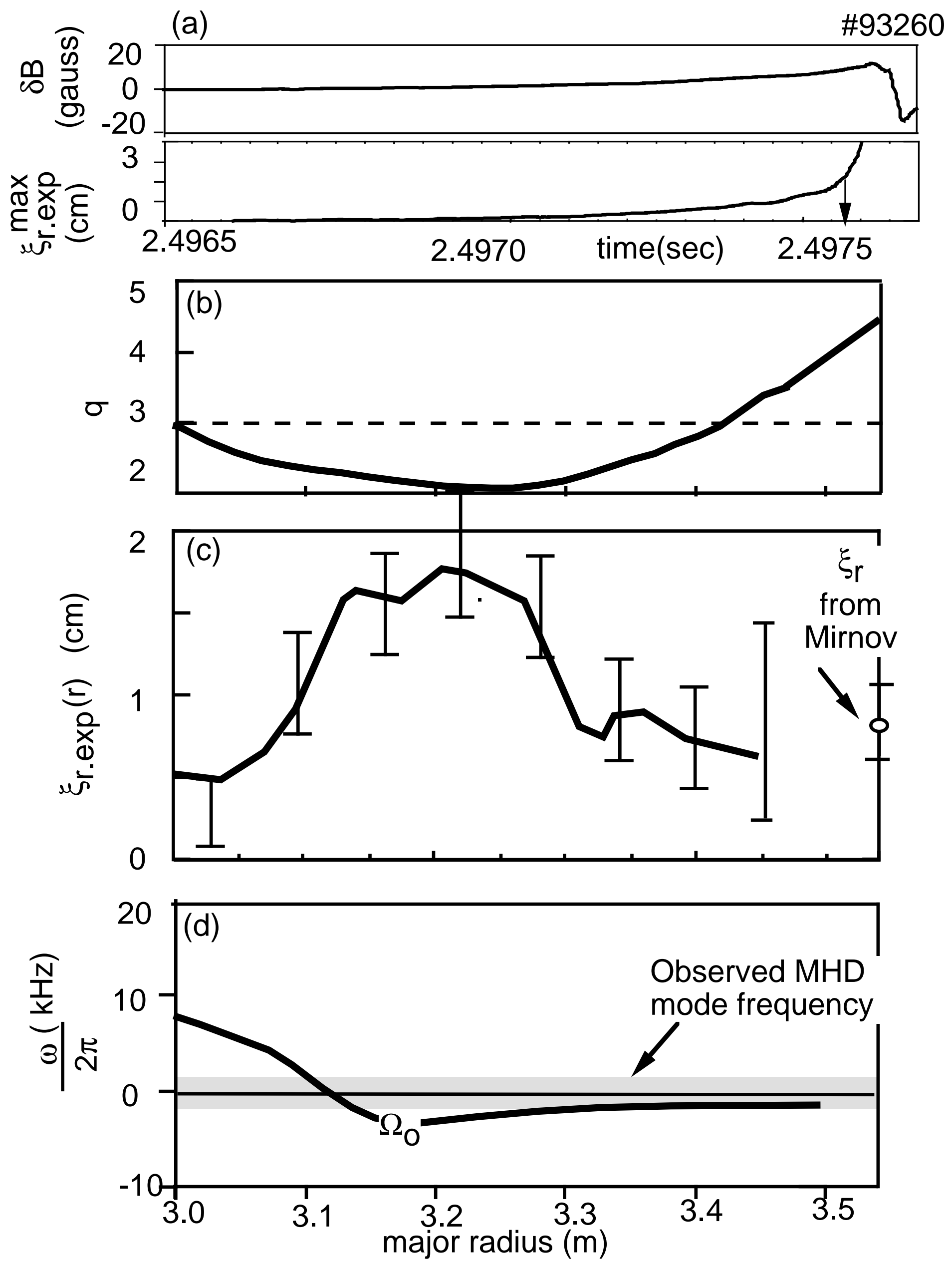

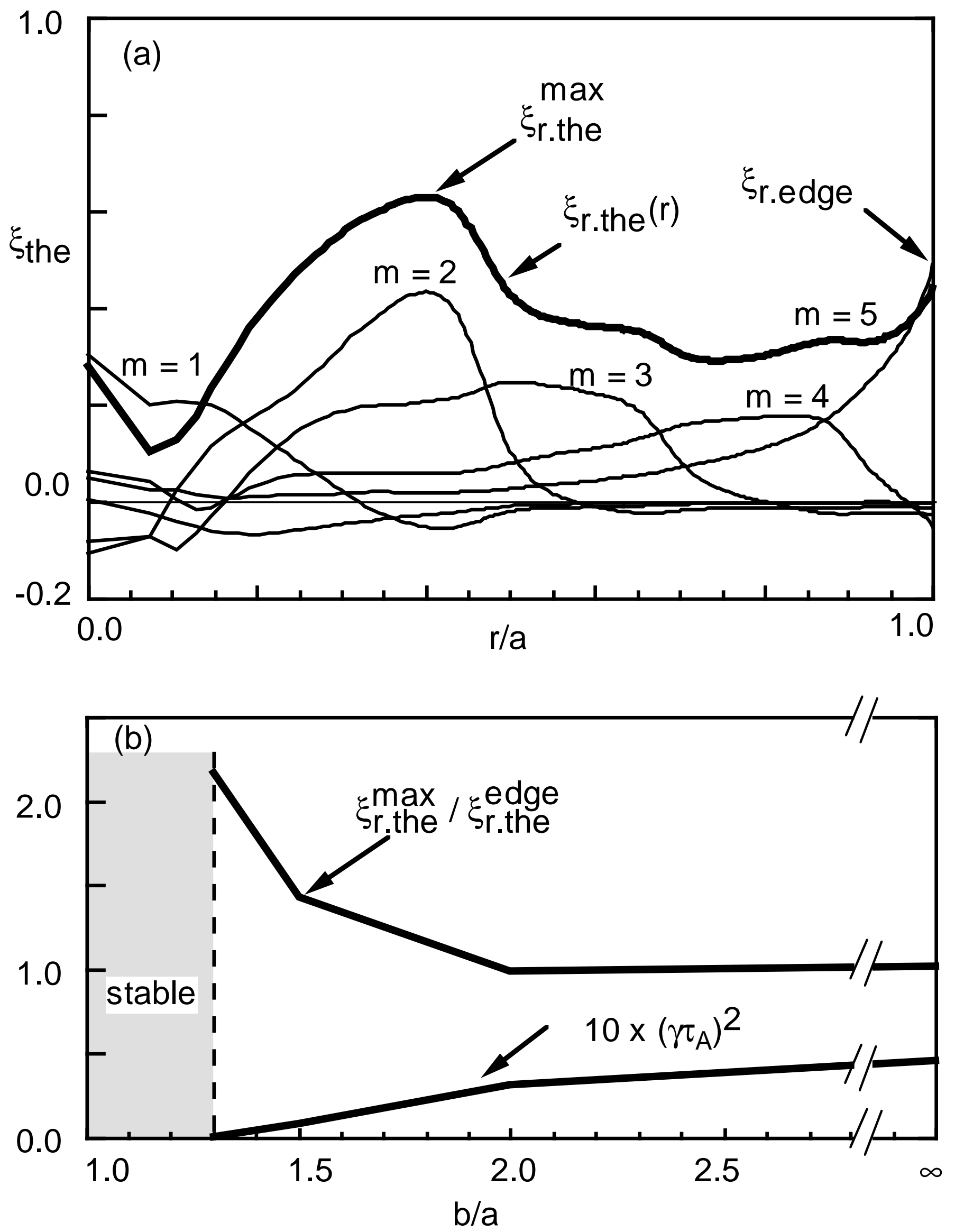

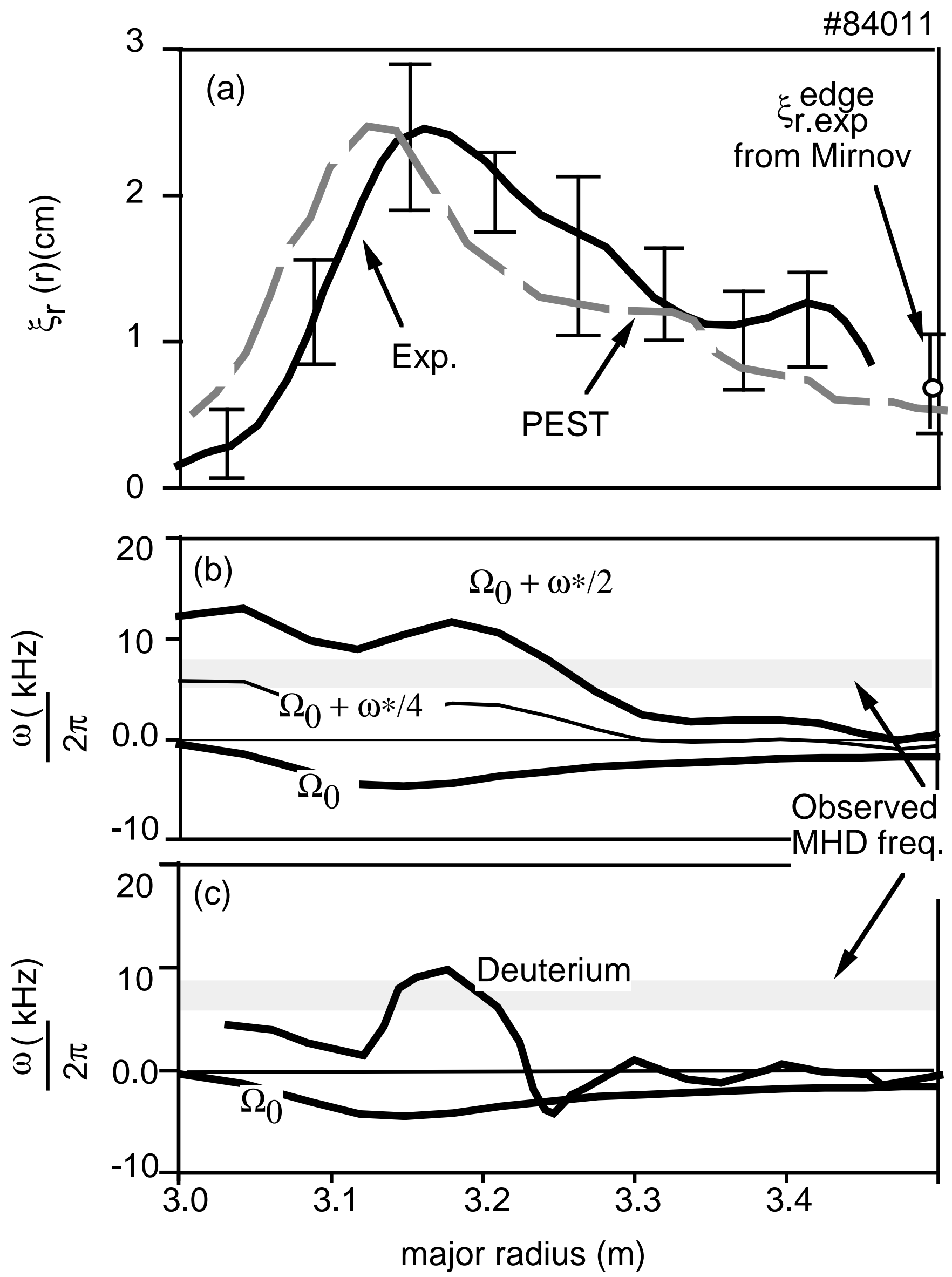

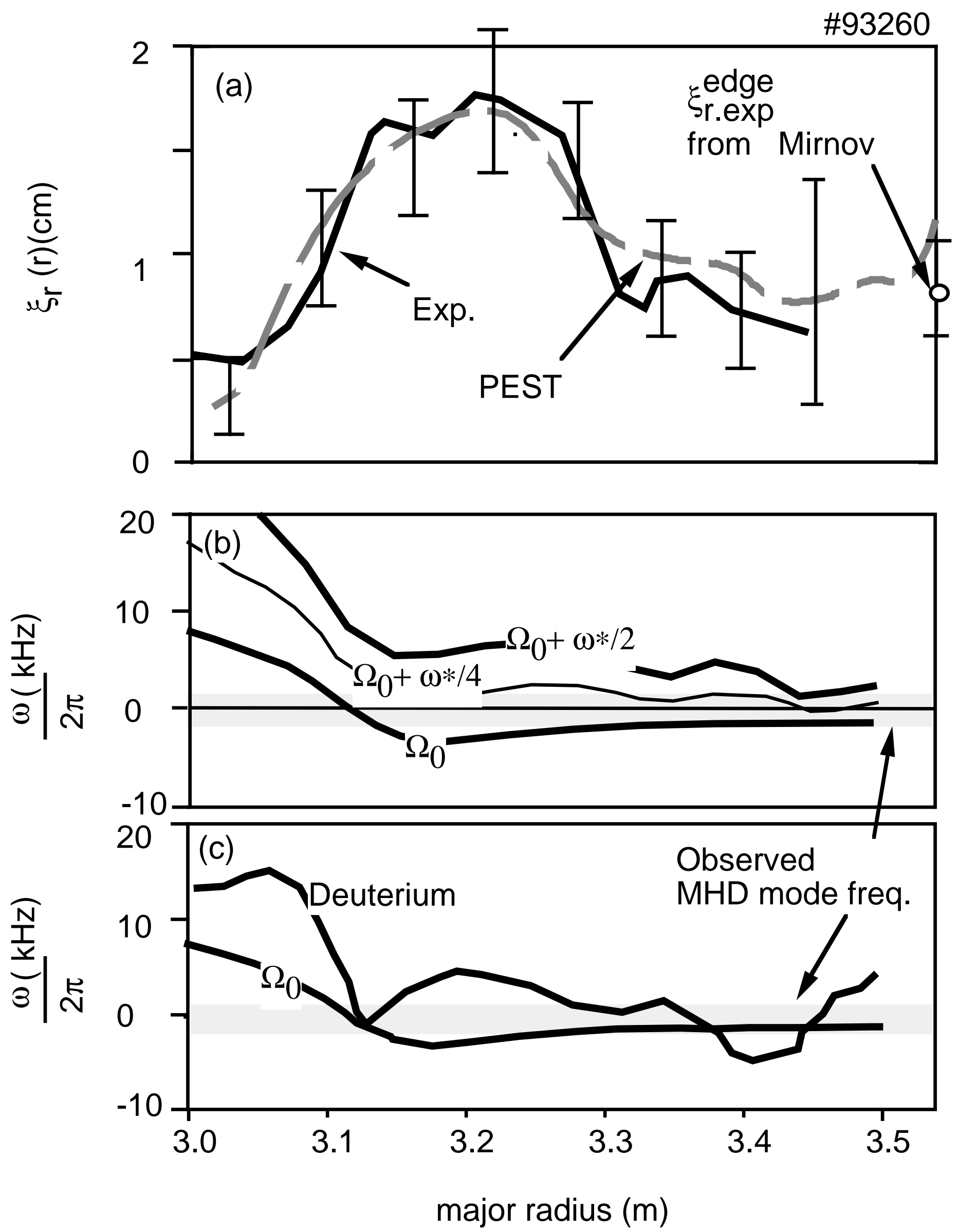


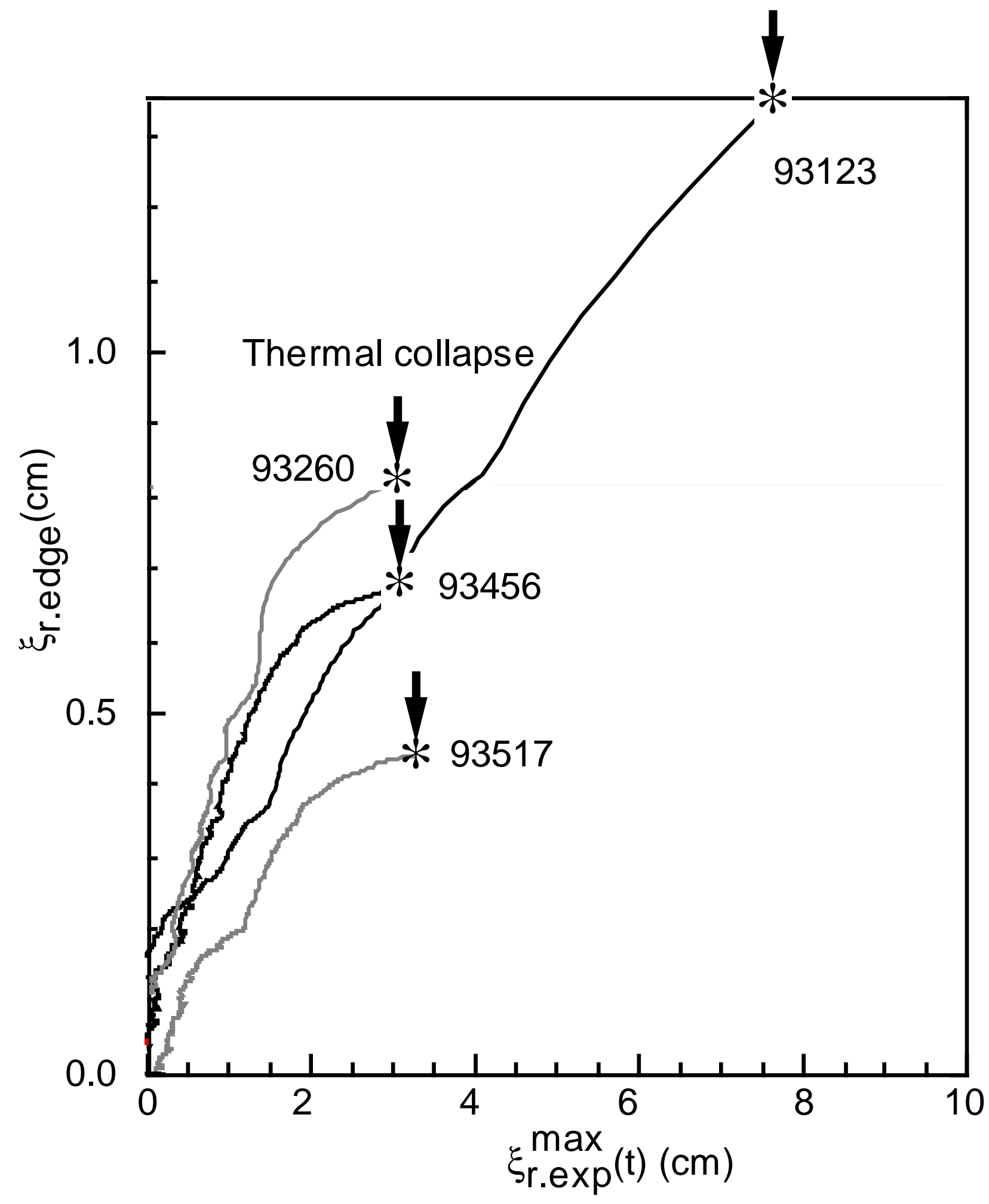

